\title{
Innovative passive multifrequency propeller device for noise and vibration reduction in turboprop fuselage
}

\author{
Angelo De Fenza ${ }^{1,3}$, Maurizio Arena ${ }^{2, *}$, and Leonardo Lecce ${ }^{2,3}$ \\ ${ }^{1}$ Department of Bioengineering and Aerospace Engineering, Universidad Carlos III de Madrid, Escuela \\ Politécnica Superior, Av. de la Universidad, 30. 28911 Leganés (Madrid), Spain \\ ${ }^{2}$ Department of Industrial Engineering - Aerospace section, University of Naples "Federico II", Via \\ Claudio, 21, Naples, 80125, Italy \\ ${ }^{3}$ NOVOTECH s.r.l. - Aerospace Advanced Technology, P.le D’Annunzio 15, 80125 Naples, Italy
}

\begin{abstract}
One of the main comfort issue affecting the passenger comfort into a turboprop aircraft fuselage is the propeller tonal noise and the related vibrations. It is well known that propeller rotation during flight generates the main noise sources, depending upon its rotational angular velocity, number of blades, power at shaft generating aircraft thrust and blades geometry. Thanks to the progress behind the control systems of the blades rotations, an innovative highly selective DVA has been conceived. The purpose of the research activity has been improving the performances of the standard passive tonal noise control system used for the BPF tuned noise and vibration attenuation in turboprop aircraft. Due to specific commercial need, the use of bi-tuned frequency can lead at a passive noise reduction at two RPM regimes. Generally, the turboprop aircrafts use only two RPM regimes: $100 \%$ at take-off, climb and approach, $86 \%$ during cruise, climb and descent. An innovative passive bi-tonal device capable to be tuned at two different frequencies in order to optimize the fuselage noise reduction at two different flight regimes (100\% and $86 \%$ ), has been designed and numerically verified. The functional effectiveness of the bi-frequential tuned device has been analysed by finite elements simulations on a linear beam, representative of the turboprop fuselage frame. The outcomes achieved within this activity encourage the advancement of this research sector, as a support to the needs of the turboprop aeronautical industry. According to the long experience gained by the research group, the proposed multifunctional concept can be a valid technology solution ready to be manufactured as well as validated in flight.
\end{abstract}

\section{Introduction}

The propeller rotation during flight generates the main noise sources depending upon its rotational angular velocity, number of blades, power at shaft generating aircraft thrust and blades geometry. The higher noise levels generated are concentrated at first Blade Passing

\footnotetext{
* Corresponding author: maurizio.arena@unina.it
} 
Frequency $\left(1^{\text {st }} \mathrm{BPF}\right)$ and its harmonics. In addition to the tuned ones, the passengers comfort can be affected also by other noise sources (Figure 1) that act with as broadband components; they are mainly linked e.g. with the blades shape, the developed engine power and the Turbulent Boundary Layer (TBL) [1-2]. These Broadband components have, generally, lower single frequency amplitude but are distribute over a wide frequency range covering all audible range, being more important at frequencies lower than $1000 \mathrm{~Hz}$.

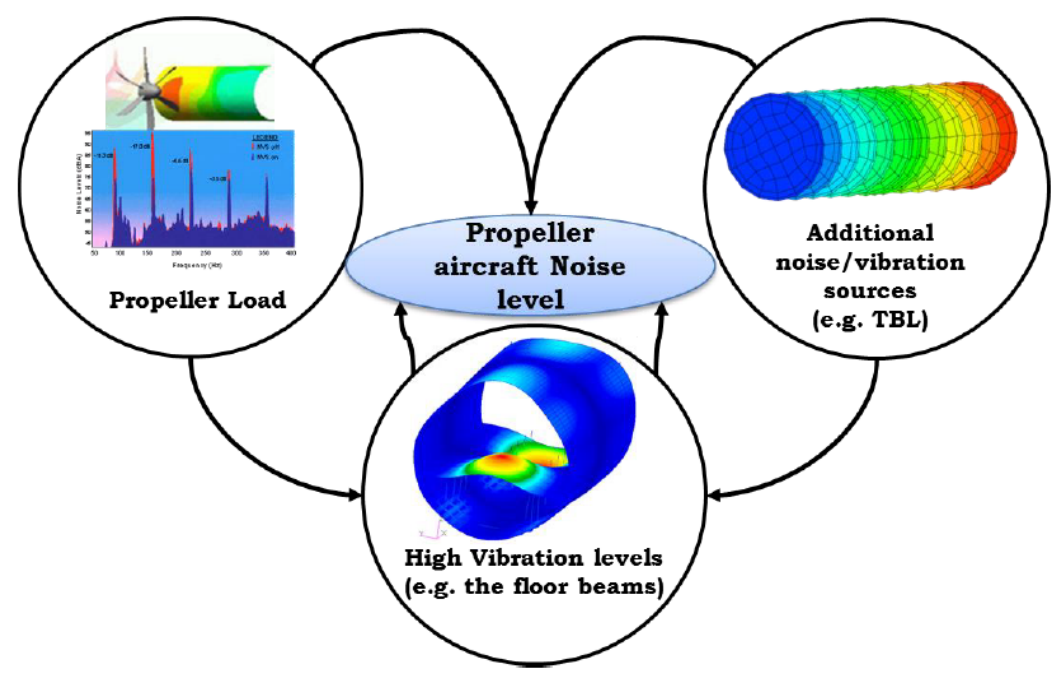

Fig. 1. Aircraft noise and vibrations sources

The needs to increase the engine power as well as the aerodynamic performances, in order to improve the aircraft performances, are such to increase the broadband noise level whole contribution up to the tonal ones thwarting the improvements reached by the use of tuned vibration absorber devices (TVA). In the same way, the need to have more performant turboprop engine in terms of stability of the thrust, have allowed the development of advanced RPM controllers able to stabilize the RPM with an accuracy, in some cases, lower than $1 \%$. This is pushing the researchers toward the development of new passive devices conceived with a different shape and working principle, with the capability to be tuned in a very narrow band (corresponding to the cruise flight condition), maximizing the efficiency [3]. An improvement of the passive devices tuned at single tone can be reached extending their functioning, to work alternatively, at two different narrow bands improving the comfort not only in cruise condition, but also to other flight regimes. As background of the whole activity, it is provided the following fundamental consideration which motivate the entire study: one of the main comfort issues affecting the passenger comfort, into a regional aircraft cabin is the propeller tonal noise with related vibrations. These design aspects have been addressed by the authors affiliation being involved by more than 30 years on propeller aircraft comfort issues, experience also gained having contributed to many research activities on the turboprop family development. Well-known are also the main commercial impacts on selling a propeller regional aircraft due to its specific noise and vibrations comfort performance capable to compete on the worldwide market. The recent innovation in RPM controller devices have changed the target related with the functionality of the passive devices for noise and vibrations control. The first vibration absorber device was applied, for first time, more than one century years ago by Watts P. W. for reducing the rolling of ship at sea [4]. The same concepts based on the use of mass-spring-damping system have been used on primary aeronautical structures subjected to external load to control the vibrations level [5-6]. When the propeller load is applied to a frequency band, the best system that allows to achieve a 
high levels of attenuation transmitted is based on the dynamic vibration absorber device. Such device, acting as damper (DVA) reduces a specific vibration of the hosting structure, usually driven by a broadband excitation. When the propeller load is concentrated to a narrow band, the dynamic vibration absorbers can be designed with a large impedance at their attachment points in a very selective range around the excitation frequency, and therefore the absorber damping is kept as small as possible. In that case it is tuned (TVA). The improvement of the engine performances, even by using RPM control devices, is opening new scenario for passive devices. The development of innovative devices with no damping materials involved, seems to be the way to improve the performances at tuned frequency, since the narrow band frequency imply a great efficiency. Such devices, based on the not utilization of damping materials (generally viscoelastic materials), have been developed by the authors [3]; conversely to the classical DVAs that work in compression, the proposed TVAs work in bending and the symmetry of the shape has been used to tune the whole device at the same frequency. Moreover, while the current DVAs are subjected to variation in behavior due to temperature effect [7-12], the TVAs realized without damping materials do not change their properties with temperature. A further development of the previous device has been done extending its work to two flight conditions characterized by different RPM regimes. In this way, in addition to the cruise flight condition, the improvement of the comfort can be obtained also to another flight condition like e.g. the climb or descent ones.

\section{Component description and validation}

As previously said, the development of innovative devices without any parts realized with damping materials is the way to improve the performances at tuned frequency $[5,6]$. The development of spring-mass devices that work in bending is the key advantage that allow to extend the operativity of the device from one to two RPM regimes. Thanks to the symmetry around its normal axis (device axisymmetric), the TVA device has two modal shapes acting perpendicularly to the surface of the frame and at the same frequency. In order to achieve the efficiency of the TVA, coupled fluid-structure simulation were performed. In particular, the effective mass of the device (Fig. 2). was evaluated numerically through design optimization analyses [13-17].

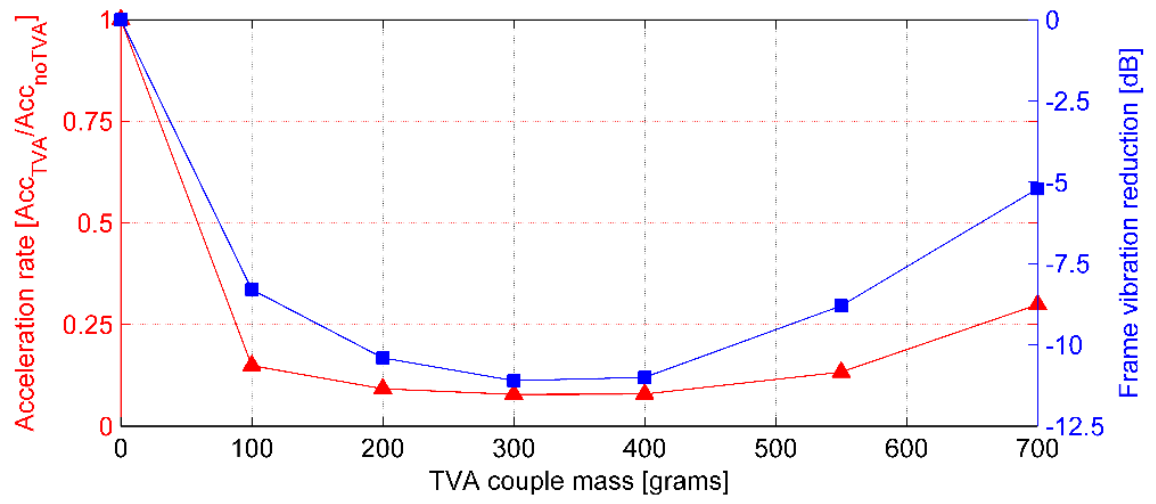

Fig. 2. Effective TVA couple mass optimization

Sensitivity analyses [18-19] were conducted to evaluate the effect of the damping of the fluid volume on the structural behavior of the fuselage portion in a coupled fluid-structure problem. Further verifications of the TVA performances (Fig. 3) in terms of fuselage cabin noise response at $1^{\text {st }} \mathrm{BPF}$ tone were performed through the vibro-acoustic approach [20-22]. 


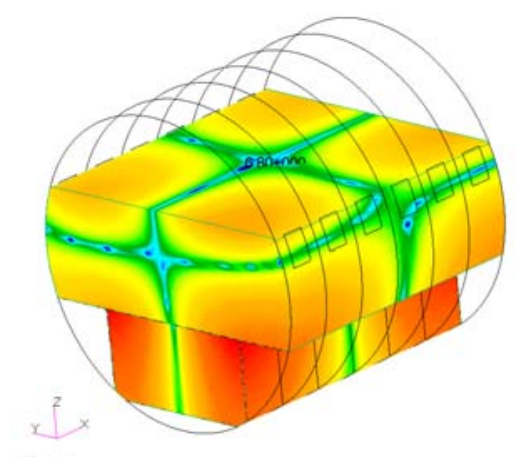

(a) Baseline
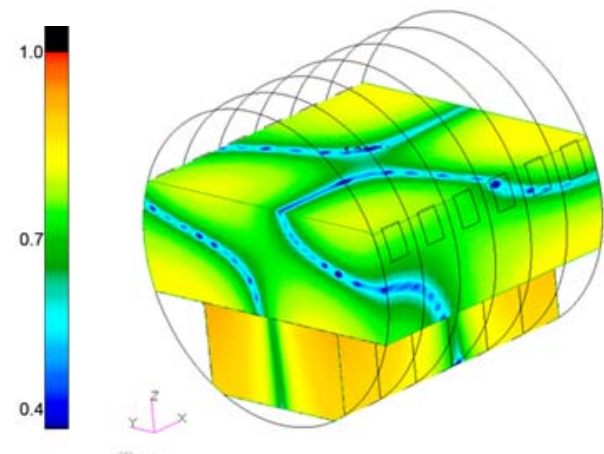

(b) TVA

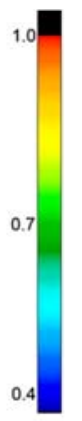

Fig. 3. Internal normalized SPL reduction assessment

In order to meet the requirement of the market that for turboprop aircrafts require an even more high comfort level, a further development to the previous device have been done in order to extend the work from one flight condition (the cruise one) to two flight conditions characterized by different RPM regimes. The improvement consists in the variation of the TVA shape such to remove the symmetry around the normal axis. In this way the TVA device has two modal shapes acting perpendicularly to the surface of the frame, but at different frequencies. The tuning frequencies of the device have been optimized to work alternatively at cruise and climb/descent flight regimes. Results in terms of vibration reduction are pictured in the Fig. 4.

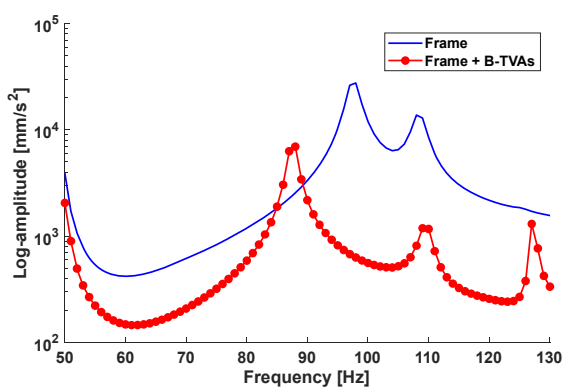

(a) First tone

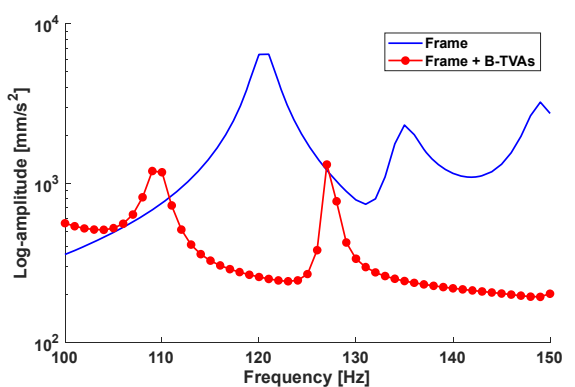

(b) Second tone

Fig. 4. Vibration attenuation of the Bi-Frequential TVA on a typical turboprop frame

\section{Conclusions and future applications}

The forecast and consequent optimization of aircraft interior noise are taking a much more central role in the design of advanced turboprop. This paper deals with the design and numerical simulations of a novel passive bi-tonal device able to be tuned at two different frequencies in order to reduce the fuselage noise with respect to two different flight regimes $(100 \%$ and $86 \%)$. The functional effectiveness of the bi-frequential tuned device has been numerically analysed considering it hosted on a linear beam, representative of the turboprop fuselage frame. The preliminary results achieved within this stage encourage the advancement of the investigation: vibration levels reduction of $32.8 \mathrm{~dB}$ and $27.9 \mathrm{~dB}$ have been respectively estimated for the two propeller tones. 


\section{References}

1. J. S. Mixson, G. C. Greene, T. K. Dempsey, NASA Technical Memorandum, 81971 (1981)

2. I. L. Ver, L. L. Beranek, Noise and Vibration Control Engineering: Principles and Applications, 2nd Edition, John Wiley \& Sons (2005)

3. M. Arena, A. De Fenza, M. Di Giulio, A. Paonessa, F. Amoroso, CEAS Aeronaut. J. 8(2), 303-312 (2017)

4. P. W. Watts, 24, 165-190 (1883)

5. V. A. Bapat, H. V. Kumaraswamy, Journal of Sound and Vibration 63(4), 469-474 (1979)

6. V. A. Bapat, P. Prabhu, Journal of Sound and Vibration 67(1), 113-119 (1979)

7. A. De Fenza, E. Monaco, F. Amoroso, L. Lecce, Journal of Vibration and Control, 22(2), 358-370, (2016)

8. M. Viscardi, M. Arena, D. Siano, Proceedings of 24th international congress on sound and vibration, ICVS24, London, UK (2017)

9. M. Viscardi, P. Napolitano, M. Arena, AIP Conference Proceedings, 1981 (2018), Article number 020100

10. M. Viscardi, M. Arena, CEAS Aeronaut J. (2018), https://doi.org/10.1007/s13272-0180326-z

11. M. Viscardi, M. Arena, D. Siano, Int. J. Mech. 10, 329-335 (2016)

12. M. Viscardi, M. Arena, D. Siano, Int. J. Mech. 10, 383-395 (2016)

13. E. Daniele, P. De Paolis, G. L. Greco, A. d'Argenio, Spatial Interaction Models. Springer Optimization and Its Applications 118. Springer, (2017)

14. P. Della Vecchia, E. Daniele, E. D’Amato, Aerospace Science and Technology 32, 103110 (2014)

15. P. Della Vecchia, L. Stingo, S. Corcione, D. Ciliberti, F. Nicolosi, A. De Marco, 18th AIAA/ISSMO Multidisciplinary Analysis and Optimization Conference, Denver, Colorado, (2017).

16. D. P. Coiro, E. Daniele, P. Della Vecchia, International Journal of Marine Energy 13, 169-179 (2016)

17. M. Viscardi, M. Arena, G. Barra, L. Guadagno, Int. J. Mech. 11, 51-57 (2017)

18. F. Amoroso, A. De Fenza, G. Petrone, R. Pecora, Archive of Mechanical Engineering 63(3), 435-454 (2016)

19. F. Amoroso, A. De Fenza, R. Pecora, P. Volpe, E. Monaco, L. Lecce, $17^{\text {th }}$ International Congress on Sound and Vibration, 2273-2280, Cairo - Egypt, (2010)

20. N. B. Roozen, Technische Universiteit Eindhoven - DOI: 10.6100/IR373471, (1992).

21. F. Amoroso, A. De Fenza, E. Monaco, R. Pecora, L. Lecce, $18^{\text {th }}$ International Congress on Sound and Vibration, 3197-3204, Rio de Janeiro - Brazil, (2011)

22. M. Viscardi, M. Arena, M. Ciminello, Proceedings Volume 10595, Active and Passive Smart Structures and Integrated Systems XII, 1059508 (2018) https://doi.org/10.1117/12.2296421 